\title{
O Rendimento do Imobiliário no Concelho de Lisboa, em Portugal
}

\author{
Real Estate Yields in the municipality of Lisbon in Portugal
}

Fernando Oliveira Tavares ${ }^{1}$; Luís Pacheco² ${ }^{2}$ Luís Gomes Almeida ${ }^{3}$
Resumo: A avaliação imobiliária pelo método do rendimento é uma atividade importante no mercado atual. Assim, este artigo tem como objetivo analisar a relação entre o valor da renda e a yield no mercado imobiliário residencial de arrendamento. Analisam-se as séries de rendas e de yields para quatro anos, o que permite realizar as comparações entre ambas através de uma análise de clusters. Este artigo estuda o valor das rendas por $\mathrm{m} 2$ para as tipologias de apartamentos T0_T1 e T2 no mercado imobiliário no concelho de Lisboa, em Portugal, para o período de 2006 a 2009. São também calculadas as yields para as diferentes tipologias, bem como uma análise de clusters para as rendas e para as yields das diferentes séries objeto de estudo. Verifica-se nesta análise que são diferentes os clusters das rendas e das yields bem como a hierarquização dos seus valores. A utilização do modelo de regressão linear neste trabalho visa estimar o valor da oferta de mercado em função da avaliação pelo método do rendimento, dos clusters das yields, da densidade populacional, entre outras. $\mathrm{Na}$ análise do mercado imobiliário é importante para o investidor racional conhecer as yields médias das diferentes zonas e como elas se agrupam em termos económico-financeiros e geográficos.

Palavras-chaves: Avaliação imobiliária; Rendimento Imobiliário; Yield Imobiliária

\begin{abstract}
The real estate evaluation by the income method is an important activity in the current market. Thus, this article aims to analyze the relationship between the rent value and the yield on residential real estate rental market. The rent and yield series for four years are analyzed, allowing comparisons between them by means of a cluster analysis. This paper studies the rents per square meter for T0_T1 and T2 apartment types in the real estate in the municipality of Lisbon - Portugal, for the period 2006 to 2009. Yields are also computed for the different apartment types, as well as a cluster analysis for rents and yields of the different series studied. In this analysis it is verified that rents and yields' clusters are different as well as the hierarchy of its values. The use of a linear regression model in this study aims to estimate the value of the market supply according to an evaluation by the income method, the clusters' yields, the population density, among others. In the housing market analysis it is important for the rational investor to know the average yields for the different areas and how they are grouped in economic, financial and geographical terms.
\end{abstract}

Keywords: Real estate evaluation, real estate income, property yield 


\section{1 - Introdução}

A utilização do modelo do rendimento na avaliação imobiliária requer a compreensão das técnicas de projeção de rendas futuras e a sua atualização. No entanto, a técnica de utilização do modelo do rendimento no âmbito do imobiliário torna-se mais arrojada do que a simples atualização dessas rendas. É necessário conhecer a "qualidade" dos contratos, a sua duração, os custos relacionados com os imóveis (a desatualização física, funcional e tecnológica é um elemento pertinente) e naturalmente as taxas de desconto. A utilização destas técnicas tem como objetivo chegar ao valor fundamental da propriedade imobiliária.

No mercado imobiliário, o processo de formação do preço permanece sujeito a comportamentos frequentemente irracionais, de tal forma que o preço de mercado pode, no curto prazo, apresentar grandes oscilações embora, a longo prazo, tenda a flutuar em torno do valor fundamental, o verdadeiro "preço" que importa aos investidores (Graham e Dodd, 1934; Gordon e Shapiro, 1956).

No método do rendimento, segundo Scolese, Bergmann, Silva e Savoia (2015), a propriedade é encarada como um bem produtivo, capaz de produzir uma renda, com determinado nível de proveito, quer se refira a um prédio urbano ou prédio rústico. $\mathrm{O}$ valor de mercado baseado na renda atual é interpretado como a soma máxima que um investidor conhecedor das atuais condições do imóvel, especialmente relacionadas com a sua ocupação presente e rendimento gerado com a renda praticada, estaria disposto a desembolsar com a sua aquisição (Sharafeddine, 2015).

Este artigo está dividido em cinco partes. Numa primeira parte é apresentada uma introdução ao tema e a apresentação do artigo em si mesmo. Numa segunda parte é efetuada uma revisão da literatura sobre o tema. Posteriormente, é apresentada a metodologia de investigação e a base de dados estudada. Na quarta parte é apresentada a análise dos valores das freguesias do concelho de Lisboa. Por fim, são apresentadas as conclusões e as limitações do estudo. 


\section{2 - Revisão da Literatura}

$\mathrm{Na}$ perspetiva do investidor, o mercado imobiliário tem interesse quando existe um retorno semelhante ao mercado acionista atendendo, naturalmente, aos princípios de rentabilidade e risco enunciados por Markowitz (1952). Assim, a perspetiva dos rendimentos futuros da propriedade imobiliária está na base da utilização do modelo do rendimento como instrumento de avaliação imobiliária.

No entender de French (2004), há várias propriedades que podem ser descritas como especiais e que deverão ser avaliadas pelo método do rendimento: terra agrícola, telecomunicações, extração mineral, bares e restaurantes, casinos e clubes, cinemas e teatros, hotéis, propriedades de lazer (privadas), casas de saúde (privadas), hospitais (privados), estações de combustíveis e bosques.

O método do rendimento identifica o valor de uma propriedade imobiliária, capaz de produzir uma renda (Molina, 2003; Rebelo, 2002, Tavares, Moreira e Pereira, 2011), sendo o seu valor dado pelo quociente entre o rendimento líquido periódico e a sua taxa de atualização. Este método é aplicável para a maior parte dos pressupostos para quem gere fundos imobiliários.

A inflação, no entender de Molina (2003), apresenta-se como um elemento crucial em todos os investimentos financeiros e também é de primordial importância em qualquer atividade relacionada com o imobiliário. No entanto, León (2003) refere que a inflação no setor imobiliário rústico é diferente da que pode ser apresentada no setor urbano e ambas são distintas da taxa de inflação geral que possa existir no mercado nacional de bens imóveis.

Molina (2003) refere ainda que quando se avalia a propriedade imobiliária, nos bens imóveis urbanos e rústicos, deve levar-se em conta o valor de reversão, entendendo como tal o valor de mercado mais favorável a que tenderia no final da sua vida útil. Da estimação desse valor fica o seguinte método: o valor de mercado do imóvel será equivalente ao custo de reversão líquido previsto no final da sua vida útil (Molina, 2003). Um dos problemas da utilização deste modelo de avaliação no mercado imobiliário é o cálculo do valor de reversão. A sua forma de cálculo pode variar, dependendo do tipo de investimento. Numa moradia com 97 anos será muito próximo do valor do terreno, deduzido dos custos de obras de demolição. Num edifício novo de escritórios poderá ser 
feito através da perpetuidade correspondente ao décimo primeiro ano e atualizada para o momento atual (Pagliari, 1991; Lusht, 2001; Hordijk, Ridder, 2005). O valor do terreno, incluindo o valor de reversão, será determinado a partir do seu valor de mercado na data da transação, o qual poderá atualizar-se com a mais ou menos valia que resulte do razoável entendimento da sua localização e uso e da evolução esperada do mercado.

Para Molina (2003) o valor de um bem é proporcional à renda económica que gera, sendo que neste tipo de avaliação é necessário avaliar o contrato de arrendamento.

$\mathrm{Na}$ avaliação pelo método do rendimento é essencial verificar a composição das rendas, a vacancy rate (e.g., taxa de desocupação) e a duração do arrendamento (Deng, Gabriel e Nothaft, 2001). A análise de equilíbrio é um instrumento precioso para a investigação de investimentos imobiliários. Ainda na avaliação imobiliária, o prémio do risco das diferentes classes de bens imóveis, o valor real da habitação e o funcionamento do mercado de arrendamento de longo prazo pode ajustar o valor da renda ao valor da avaliação.

Black, Fraser e Hoesli (2006) entendem que o método do rendimento permite examinar a relação entre os valores fundamentais, preços de mercado e bolhas especulativas no mercado habitacional. A cap rate, que representa o retorno anual de um investimento antes dos pagamentos da hipoteca e do imposto sobre o rendimento, está relacionada com o valor futuro das rendas dos bens imóveis (Ghysels, Plazzi e Valkanov, 2006).

O método do rendimento, utilizado essencialmente para avaliar as propriedades atrás referidas, apresenta vantagens pois permite verificar a compatibilidade entre os preços de mercado e os valores fundamentais (Ho, Rengarajan, Glascock, 2014; Tavares e Almeida, 2015; Gallin, 2003). Esta configuração traz vantagens para determinadas entidades financeiras, pois é aplicável para a maior parte dos pressupostos de quem gere fundos imobiliários, onde as rendas se apresentam como um aspeto importante (Molina, 2003).

Moreira, Tavares e Pereira (2014) concluíram que neste método a utilização de uma cap rate na previsão dos retornos é economicamente significativa e estimaram o valor da propriedade imobiliária como o valor atual das suas rendas futuras. French (2004) defende que, para a maioria das propriedades comuns, o valor da propriedade está baseado na sua renda potencial, vista como um investimento. Neste contexto, Grenadier (2003) e Tabner 
(2007) referiram que o método do rendimento permite verificar o valor fundamental e comparar com o mercado.

Embora a proximidade e o valor sentimental sejam aspetos relevantes no mercado imobiliário, eles não são de facto os valores que interessam aos investidores. Vários autores referem que o valor atual também pode ser aplicado ao mercado imobiliário (Campbell, 1987; Campbell e Shiller, 1987; Meese e Wallace, 1993). Por sua vez De Long et al (1990) verificam a existência de mispricing. Para os autores anteriormente referidos, o valor de um bem é proporcional à renda que ele gera ou pode vir a gerar no futuro.

A utilização do método do rendimento apresenta alguns inconvenientes. Para Ghysels, Plazzi e Valkanov (2006) o cálculo do valor da propriedade imobiliária, como qualquer outro recurso financeiro descontando o valor das rendas esperadas, não tem tido muita aceitação no mundo académico. Molina (2003) refere que o método apresenta vários elementos de difícil quantificação: o valor de reversão, a vida útil, as mais-valias e as menos-valias. Também Wang e Zhou (2000) expõem a dificuldade no conhecimento do rendimento futuro da propriedade, da duração desses rendimentos e da conjuntura económica. O método do rendimento apresenta dificuldade na decisão da taxa de desconto (Geltner et al 2007; Clayton, Ling e Naranjo, 2009).

Na previsão de rendas futuras, Pagliari (1991), Lusht (2001) e Hordijk, Ridder (2005) entendem que o avaliador deveria usar o décimo primeiro ano como o primeiro ano de exploração para o comprador. Boyd (2002) também entende que o décimo primeiro ano também deveria ser utilizado. Van Gool, Jager e Weisz (2001) advertem para o facto de o décimo ou décimo primeiro anos serem mitigados, pois há custos de manutenção elevados ou custos extra de mudança de inquilinos.

De acordo com Lusht (2001) e Van Gool, Jager e Weisz (2001), os avaliadores deveriam descontar aos compradores os custos, pois caso contrário, a pessoa inclui custos que deveriam ter um tratamento fiscal diferente. Para os autores anteriormente referidos os dez anos são vistos como os mais apropriados. Isto porque, aumentando os períodos para quinze anos, as previsões começam a ser complicadas e os resultados refletem cenários artificiais com pouca aderência à realidade. Por outro lado, se o período é pequeno, 
entenda-se três ou sete anos, o impacto do valor residual é elevado o que faz com que os cash flows sejam inferiores.

\section{3 - Metodologia de Investigação}

Para a análise da aderência à realidade portuguesa da avaliação imobiliária, do modelo do rendimento, foi utilizado o modelo da perpetuidade referido por de Brown e Cliff (2005), Geltner et al (2007) e Clayton, Ling e Naranjo (2009) e a sua relação entre os valores fundamentais e os valores sentimentais da avaliação imobiliária.

Para Clayton, Ling e Naranjo (2009) o preço de equilíbrio da propriedade no período t, $\mathrm{P}_{\mathrm{t}}$, deve ser igual ao valor atual dos Net Operating Income (NOI) descontados, assumindo uma taxa de risco ajustada, constante e não alavancada, $r_{t}$. Isto é,

$$
\begin{aligned}
P_{t}^{e}=\frac{N O I_{1}}{\left(1+r_{t}\right)}+ & \frac{N O I_{2}}{\left(1+g_{t=2}\right)}+\frac{N O I_{3}\left(1+g_{t=3}\right)}{\left(1+r_{t}\right)^{3}} \\
& +\ldots \frac{N O I_{T-1}\left(1+g_{t=T}\right)+N S P_{T}}{\left(1+r_{t}\right)^{T}}
\end{aligned}
$$

- T é o período esperado de exploração em anos

- $\quad \mathrm{NSP}_{\mathrm{T}}$ é a esperada venda líquida do produto no ano $\mathrm{T}^{1}$

- NOI são as receitas líquidas esperadas

Para Geltner et al (2007) é possível simplificar a fórmula anterior, isto porque o NOI prevê um crescimento constante e sendo o $\mathrm{P}_{\mathrm{t}}^{\mathrm{e}}$ a função do crescimento esperado do NOI para uma determinada taxa de risco e propriedade específica. Isto é:

$$
P_{t}^{e}=\frac{N O I_{1}}{r_{t}-g}=\frac{N O I_{1}}{R_{t}^{e}} \text { ou }-\frac{P_{\underline{t}}^{e}}{N O I_{1}}=\frac{1}{r_{t}-g}
$$

- Rt é a cap rate de equilíbrio no momento t;

- gt taxa de crescimento do NOI no momento $t$ (espera-se constante).

Normalmente, a taxa de crescimento corresponde à valorização do imóvel

$$
\text { - } R_{t}^{e}=r_{t}-g_{t}
$$

\footnotetext{
${ }^{1}$ No NOI é assumido que inclui uma reserva para gastos de capital e outras despesas esperadas tais como leasing de comissões.
} 
De notar que os valores do imóvel podem ser expressos como múltiplos do primeiro ano NOI de dimensão múltipla da função, da taxa de desconto específica da propriedade e das mudanças esperadas no $\mathrm{NOI}^{2}$.

Neste trabalho, a avaliação pelo método do rendimento foi efetuada utilizando uma taxa de desconto calculada através do modelo do Capital Asset Pricing Model (CAPM).

$$
E\left(R_{I}\right)=R_{f}+\beta_{i}\left(E\left(R_{m}\right)-R_{f}\right)
$$

Onde:

- $\quad E\left(R_{i}\right)$ é o custo do capital;

- $\quad R_{f}$ a taxa isenta de risco;

- $E\left(R_{m}\right)$ a esperança de rentabilidade do mercado;

- O O $\beta_{\mathrm{i}}$ é o beta das tipologias T0_T1 e T2.

O Capital Asset Pricing Model (CAPM) foi desenvolvido por Sharpe (1964), Lintner (1965) e Mossin (1966) a partir das conclusões do trabalho de Markowitz (1959).

O beta foi calculado pela fórmula:

$$
\beta_{i}=\frac{\operatorname{Cov}\left(R_{i}, R_{m}\right)}{\operatorname{Var}\left(R_{m}\right)}
$$

Onde:

- $\operatorname{Cov}\left(R_{i}, R_{m}\right)$ representa a covariância entre a rentabilidade dos T0_T1 e T2 e a rentabilidade do mercado;

- $\operatorname{Var}\left(R_{m}\right)$ representa a variância da rentabilidade do mercado.

Como $R_{f}$ foi utilizada a série da taxa de rentabilidade de Obrigações do Tesouro a taxa fixa, por prazo de 2 anos. A rentabilidade do mercado é dada pelos valores médios das yields para o trimestre correspondente, tendo sido calculado em separado para os T0_T1 e para os T2.

O beta foi calculado através da fórmula atrás referida. O beta dos T0_T1 é de 1,055 e o da tipologia T2 de 1,036. O beta também é uma medida de risco, pelo que podemos concluir que o risco dos T0_T1 é mais elevado que o dos T2.

Também foram calculadas as yields dos concelhos e freguesias para os valores apresentados na amostra. $\mathrm{O}$ valor da yield é calculado dividindo o valor do rendimento

\footnotetext{
${ }^{2}$ Alterações nos impostos e taxas que afetam bens e valores, por isso, Preço/NOI é múltiplo, com montantes diversos e custo de financiamento e a hipoteca.
} 
pelo valor do preço de oferta (Ling e Archer, 2006). Segundo Laia (2007), a fórmula da yield ou cap rate pode escrever-se da seguinte forma:

$$
V_{0}=\frac{R_{1}}{y_{1}}
$$

Em que:

- V $\quad V_{0}$ é o valor do imóvel;

- $\quad R_{1}$ é o rendimento previsto para o primeiro ano de exploração;

- $\quad Y_{1}$ é a Cap Rate ou yield inicial.

Assim, conhecendo um determinado imóvel e a cap rate verificada no mercado para imóveis semelhantes, esta fórmula pode ser utilizada para calcular o seu valor. Trata-se da fórmula da perpetuidade sem crescimento, que pressupõe que o rendimento do imóvel se mantém sempre igual e constante no futuro.

No presente artigo é efetuada uma análise de clusters para os valores das rendas e das yields das freguesias de Lisboa, em Portugal. A análise de clusters é uma técnica exploratória de análise multivariada que tem como objetivo o agrupamento de sujeitos ou variáveis com base nas suas características. Nesta análise, os dados agrupados no mesmo Cluster são muito semelhantes relativamente a qualquer um dos critérios de seleção predeterminado (Pestana e Gageiro, 2014; Maroco, 2014). Cada observação pertencente a um determinado cluster é similar a todas as outras pertencentes a esse cluster, e é diferente das observações pertencentes aos demais clusters. Segundo Maroco (2014) não existe um melhor "critério" de agregação hierárquica e o autor recomenda a utilização de vários métodos em simultâneo, sendo que, se produzirem soluções interpretáveis similares se pode concluir que se trata de agrupamentos "naturais" e não "artefactuais". Importa referir que foram utilizados os vários critérios de agregação hierárquica com a finalidade de cumprir o objetivo de as diferenças entre clusters serem estatisticamente significativas.

A análise de regressão pode ser usada para modelar a relação funcional entre duas variáveis (Gujarati, 2003). Através da regressão linear múltipla analisa-se a relação entre a variável dependente (valor da oferta de mercado) e um conjunto de variáveis explicativas. O modelo tem como variável dependente o valor da oferta de mercado e como variáveis independentes a avaliação efetuada através do modelo de Clayton, Ling e Naranjo (2009), tendo sido utilizada como taxa de desconto a calculada através do CAPM, os clusters das yields (como variáveis dummy) e a densidade populacional. De 
referir que outras variáveis foram testadas nomeadamente a taxa de atualização de rendas e a taxa de crescimento do Produto Interno Bruto (PIB), mas rejeitadas por não apresentarem resultados estatisticamente satisfatórios. $O$ modelo de regressão na população será:

$$
Y=\beta 0+\beta 1 X 1+\beta 2 X 2+\cdots+\beta K X K+u
$$

Onde:

- $\quad$ B's são os coeficientes, que quantificam a variação que uma variável explicativa tem sobre a variável dependente;

- Os X's, representam as variáveis explicativas e Y a variável dependente, u é o resíduo que traduz a diferença entre $\mathrm{Y}$ e o seu valor esperado. Note-se que há variáveis Xi que são variáveis Dummy que podem ter alternativamente o valor "0" ou " 1 ".

\section{1 - Base de Dados para Estudo}

Para o estudo empírico foi usada a base de dados das séries estatísticas da Imométrica para os anos de 2006, 2007, 2008 e 2009. Foram utilizadas as séries trimestrais de dados dos valores por metro quadrado dos apartamentos usados apresentados em oferta no concelho de Lisboa. Os apartamentos sobre os quais incidiu o estudo foram a série dos T0 e T1 e T2. Para construir a série de dados trimestrais foram calculados os valores médios de oferta por metro quadrado nas tipologias T0_T1 e T2.

De forma a homogeneizar os dados obtidos e evitar outliers foi decidido retirar da base de dados os imóveis que: (a) a nível de preços apresentavam valores de renda mensal superior a $25000 €$ e inferiores a 50€; (b) a nível de áreas apresentavam valores superiores a $500 \mathrm{~m}^{2}$ e inferiores a $10 \mathrm{~m}^{2}$; e (c) a nível de renda por $\mathrm{m}^{2}$ apresentavam valores de renda mensal superior a $50 € / \mathrm{m}^{2}$ e inferiores a $2 € / \mathrm{m}^{2}$.

Resultaram desta metodologia séries para as tipologias T0_T1 (agrupadas numa só série) e para a tipologia $\mathrm{T} 2$.

Relativamente às taxas de desconto calculadas com base no CAPM (referido anteriormente) foram as seguintes para os apartamentos T0_T1 e para os T2:

Quadro 1 - Cálculo dos valores das taxas de desconto dos apartamentos T0_T1 pelo CAPM
\begin{tabular}{|c|c|c|c|c|}
\hline T0_T1 & RF & Mercado & Beta & CAPM \\
\hline $1^{\circ}$ Trimestre_2006 & 3,13 & 5,36 & 1,055 & $5,48 \%$ \\
\hline $2^{\circ}$ Trimestre_2006 & 3,35 & 5,44 & 1,055 & $5,55 \%$ \\
\hline
\end{tabular}




\begin{tabular}{|l|l|l|l|l|}
\hline $3^{\circ}$ Trimestre_2006 & 3,66 & 5,43 & 1,055 & $5,52 \%$ \\
\hline $4^{\circ}$ Trimestre_2006 & 3,80 & 5,45 & 1,055 & $5,54 \%$ \\
\hline $1^{\circ}$ Trimestre_2007 & 4,02 & 5,22 & 1,055 & $5,29 \%$ \\
\hline $2^{\circ}$ Trimestre_2007 & 4,51 & 5,38 & 1,055 & $5,43 \%$ \\
\hline $3^{\circ}$ Trimestre_2007 & 4,13 & 5,48 & 1,055 & $5,56 \%$ \\
\hline $4^{\circ}$ Trimestre_2007 & 4,10 & 5,51 & 1,055 & $5,59 \%$ \\
\hline $1^{\circ}$ Trimestre_2008 & 3,63 & 5,33 & 1,055 & $5,42 \%$ \\
\hline $2^{\circ}$ Trimestre_2008 & 4,79 & 5,28 & 1,055 & $5,31 \%$ \\
\hline $3^{\circ}$ Trimestre_2008 & 4,24 & 5,37 & 1,055 & $5,43 \%$ \\
\hline $4^{\circ}$ Trimestre_2008 & 2,84 & 5,60 & 1,055 & $5,75 \%$ \\
\hline $1^{\circ}$ Trimestre_2009 & 2,31 & 5,77 & 1,055 & $5,96 \%$ \\
\hline $2^{\circ}$ Trimestre_2009 & 1,80 & 5,80 & 1,055 & $6,02 \%$ \\
\hline $3^{\circ}$ Trimestre_2009 & 1,27 & 5,76 & 1,055 & $6,01 \%$ \\
\hline $4^{\circ}$ Trimestre_2009 & 1,30 & 5,75 & 1,055 & $6,00 \%$ \\
\hline
\end{tabular}

Fonte: Elaboração própria

\begin{tabular}{|c|c|c|c|c|}
\hline $\mathrm{T} 2$ & $\mathrm{RF}$ & Mercado & Beta & CAPM \\
\hline $1^{\circ}$ Trimestre_2006 & 3,13 & 5,02 & 1,036 & $5,09 \%$ \\
\hline $2^{\circ}$ Trimestre_2006 & 3,35 & 4,90 & 1,036 & $4,96 \%$ \\
\hline $3^{\circ}$ Trimestre_2006 & 3,66 & 4,94 & 1,036 & $4,99 \%$ \\
\hline $4^{\circ}$ Trimestre_2006 & 3,80 & 5,00 & 1,036 & $5,05 \%$ \\
\hline $1^{\circ}$ Trimestre_2007 & 4,02 & 4,72 & 1,036 & $4,74 \%$ \\
\hline $2^{\circ}$ Trimestre_2007 & 4,51 & 5,13 & 1,036 & $5,16 \%$ \\
\hline $3^{\circ}$ Trimestre_2007 & 4,13 & 5,21 & 1,036 & $5,25 \%$ \\
\hline $4^{\circ}$ Trimestre_2007 & 4,10 & 5,39 & 1,036 & $5,44 \%$ \\
\hline $1^{\circ}$ Trimestre_2008 & 3,63 & 5,12 & 1,036 & $5,18 \%$ \\
\hline $2^{\circ}$ Trimestre_2008 & 4,79 & 5,12 & 1,036 & $5,13 \%$ \\
\hline $3^{\circ}$ Trimestre_2008 & 4,24 & 5,14 & 1,036 & $5,17 \%$ \\
\hline $4^{\circ}$ Trimestre_2008 & 2,84 & 5,50 & 1,036 & $5,60 \%$ \\
\hline $1^{\circ}$ Trimestre_2009 & 2,31 & 5,30 & 1,036 & $5,41 \%$ \\
\hline $2^{\circ}$ Trimestre_2009 & 1,80 & 5,26 & 1,036 & $5,39 \%$ \\
\hline $3^{\circ}$ Trimestre_2009 & 1,27 & 5,25 & 1,036 & $5,39 \%$ \\
\hline $4^{\circ}$ Trimestre_2009 & 1,30 & 5,28 & 1,036 & $5,42 \%$ \\
\hline
\end{tabular}

Fonte: Elaboração própria

\section{4 - Análise dos Valores das Freguesias de Lisboa}

O Quadro 3 apresenta os valores por $\mathrm{m}^{2}$ médios das rendas das freguesias do concelho de Lisboa que apresentavam dados disponíveis para o período de 2006-2009, dos apartamentos T0_T1e T2. Nos apartamentos da tipologia T0_T1, os valores médios da renda em $€ / \mathrm{m}^{2}$ mais elevados são apresentados pelas rendas das freguesias de São Sebastião da Pedreira com 14,15€, da Encarnação com 13,97€ e Coração de Jesus com $13,47 €$, enquanto para a tipologia T2 os valores mais elevados são ostentados pelas freguesias de São Sebastião da Pedreira com 12,74€, Encarnação com 12,59€ e Santa Isabel com 12,38€. 
Os valores médios de renda por $\mathrm{m}^{2}$ mais baixos são apresentados, para as tipologias T0_T1, pela freguesia do Beato com 9,43€, São Vicente de Fora com 10,54€ e Santa Engrácia com 10,56€. Para a tipologia T2 os valores mais baixos são apresentados pelas freguesias de S. João e Penha de França com 8,67€ e Anjos com 8,69€. O valor médio das rendas por $\mathrm{m}^{2}$ para as tipologias T0_T1 para as freguesias apresentadas é de 11,91€ e para a tipologia T2€ para o mesmo período é de 10,28€. Assim, o valor médio das rendas dos T0_T1 é superior em 15\% ao valor das rendas dos T2.

Relativamente às rendas das freguesias do concelho de Lisboa a solução mais fiável e consistente encontrada baseia-se na formação de três clusters de variáveis tendo como método de agregação o método centroid linkage e como medida de agregação a distância euclidiana, conforme é possível observar no Quadro 3.

Quadro 3 - Média $(€ / \mathrm{m} 2)$ e desvio padrão das rendas dos dados das freguesias do concelho de Lisboa

\begin{tabular}{|c|c|c|c|c|c|c|c|c|c|}
\hline \multicolumn{5}{|c|}{ Freguesias de Lisboa - Tipologias T0 e T1 } & \multicolumn{5}{|c|}{ Freguesias de Lisboa - Tipologias T2 } \\
\hline & Média & $\begin{array}{l}\text { Desvio } \\
\text { Padrão }\end{array}$ & Cluster & $\begin{array}{c}\text { Renda } \\
\text { Média do } \\
\text { Cluster }\end{array}$ & & Média & $\begin{array}{l}\text { Desvio } \\
\text { Padrão }\end{array}$ & Cluster & $\begin{array}{l}\text { Renda } \\
\text { Média do } \\
\text { Cluster }\end{array}$ \\
\hline S. Sebastião Pedreira & 14,15 & 1,59 & \multirow{2}{*}{3} & \multirow{2}{*}{14,06} & S. Sebastião Pedreira & 12,74 & 1,17 & \multirow{7}{*}{3} & \multirow{7}{*}{12,08} \\
\hline Encarnação & 13,97 & 2,51 & & & Encarnação & 12,59 & 1,89 & & \\
\hline Coração de Jesus & 13,47 & 1,65 & \multirow{34}{*}{2} & \multirow{34}{*}{11,85} & Santa Isabel & 12,38 & 2,03 & & \\
\hline São João de Deus & 13,40 & 1,25 & & & S. Maria de Belém & 11,91 & 0,73 & & \\
\hline São Mamede & 13,18 & 1,89 & & & S. Maria Olivais & 11,82 & 0,84 & & \\
\hline Mercês & 13,07 & 1,40 & & & Coração de Jesus & 11,63 & 1,30 & & \\
\hline Santa Maria Olivais & 13,05 & 0,91 & & & São José & 11,52 & 1,90 & & \\
\hline São José & 12,94 & 2,22 & & & Pena & 11,27 & 0,69 & \multirow{10}{*}{2} & \multirow{10}{*}{10,73} \\
\hline Santa Catarina & 12,74 & 1,61 & & & São Mamede & 11,22 & 0,55 & & \\
\hline Campolide & 12,65 & 0,93 & & & Campolide & 11,16 & 1,38 & & \\
\hline N. Senhora Fátima & 12,33 & 1,00 & & & Mercês & 11,02 & 1,46 & & \\
\hline Campo Grande & 12,31 & 1,07 & & & Lapa & 10,74 & 0,80 & & \\
\hline Santa Isabel & 12,05 & 1,22 & & & Campo Grande & 10,50 & 0,99 & & \\
\hline São Jorge Arroios & 12,04 & 1,18 & & & N. Senhora Fátima & 10,47 & 0,81 & & \\
\hline Lapa & 12,00 & 0,88 & & & S. João de Deus & 10,33 & 1,83 & & \\
\hline S. João de Brito & 11,92 & 0,68 & & & São Jorge de Arroios & 10,29 & 1,20 & & \\
\hline Prazeres & 11,88 & 1,00 & & & Santos-o-Velho & 10,29 & 1,10 & & \\
\hline S. Domingos Benfica & 11,84 & 0,65 & & & Alvalade & 10,19 & 1,11 & \multirow{20}{*}{1} & \multirow{20}{*}{9,43} \\
\hline Pena & 11,77 & 2,35 & & & Prazeres & 10,12 & 1,20 & & \\
\hline S. Francisco Xavier & 11,76 & 1,05 & & & S. Francisco Xavier & 10,03 & 0,85 & & \\
\hline Santo Condestável & 11,66 & 0,83 & & & Santo Condestável & 10,00 & 0,74 & & \\
\hline Alcântara & 11,65 & 1,09 & & & S. João de Brito & 9,95 & 0,77 & & \\
\hline Carnide & 11,59 & 0,86 & & & Santa Catarina & 9,91 & 1,42 & & \\
\hline São João & 11,44 & 1,84 & & & Graça & 9,86 & 1,62 & & \\
\hline Graça & 11,35 & 1,21 & & & Alto do Pina & 9,75 & 1,44 & & \\
\hline Alto do Pina & 11,29 & 0,78 & & & Carnide & 9,53 & 0,80 & & \\
\hline S. Maria Belém & 11,25 & 1,42 & & & S. Domingos Benfica & 9,44 & 0,48 & & \\
\hline Alvalade & 11,24 & 1,10 & & & Benfica & 9,39 & 0,62 & & \\
\hline Lumiar & 11,24 & 0,89 & & & Lumiar & 9,32 & 0,59 & & \\
\hline Penha de França & 11,16 & 0,64 & & & Santa Engrácia & 9,22 & 0,80 & & \\
\hline Ajuda & 11,14 & 1,73 & & & Alcântara & 9,20 & 0,71 & & \\
\hline Santos-o-Velho & 10,95 & 1,31 & & & S. Vicente de Fora & 8,95 & 0,91 & & \\
\hline Anjos & 10,90 & 1,40 & & & Ajuda & 8,91 & 0,75 & & \\
\hline Benfica & 10,63 & 0,82 & & & Beato & 8,77 & 0,73 & & \\
\hline Santa Engrácia & 10,56 & 1,29 & & & Anjos & 8,69 & 1,21 & & \\
\hline S. Vicente de Fora & 10,54 & 2,34 & & & Penha de França & 8,67 & 1,19 & & \\
\hline Beato & 9,43 & 1,24 & 1 & 9,43 & S. João & 8,67 & 0,64 & & \\
\hline Média & 11,91 & 1,29 & & & Média & 10,28 & 1,06 & & \\
\hline
\end{tabular}

Fonte: Elaboração própria

Assim são formados 2 conjuntos de variáveis com as rendas médias para os T0_T1 sendo eles: 
- Cluster 1 - Beato, sendo a renda média do cluster de $9,43 € / \mathrm{m}^{2}$;

- Cluster 2 - Coração de Jesus, São João de Deus, São Mamede, Mercês, Santa Maria Olivais, São José, Santa Catarina, Campolide, N. Senhora Fátima, Campo Grande, Santa Isabel, São Jorge Arroios, Lapa, S. João de Brito, Prazeres, S. Domingos Benfica, Pena, S. Francisco Xavier, Santo Condestável, Alcântara, Carnide, São João, Graça, Alto do Pina, S. Maria Belém, Alvalade, Lumiar, Penha de França, Ajuda, Santos-o-Velho, Anjos, Benfica, Santa Engrácia, S. Vicente de Fora, com rendas a variar de 13,47€ a 10,54€/m² e um valor médio de cluster de $11,85 € / \mathrm{m}^{2}$.

- Cluster 3 - S. Sebastião Pedreira e Encarnação, com rendas a variar de $14,15 €$ a $13,97 € / \mathrm{m}^{2}$ e um valor médio de cluster de $14,06 € / \mathrm{m}^{2}$.

As diferenças entre os clusters 1, 2 e 3 são estatisticamente significativas a 95\%.

Quanto às rendas para os apartamentos T2 encontrou-se uma solução de três clusters com o recurso ao método ward method, tendo como medida de agregação a distância euclidiana quadrada, conforme é possível observar no Quadro 3.

Os três clusters formados são assim:

- Cluster 1 - Alvalade, Prazeres, S. Francisco Xavier, Santo Condestável, S. João de Brito, Santa Catarina, Graça, Alto do Pina, Carnide, S. Domingos Benfica, Benfica, Lumiar, Santa Engrácia, Alcântara, S. Vicente de Fora, Ajuda, Beato, Anjos, Penha de França e S. João, cujos valores das rendas estão compreendidos entre $10,19 €$ e $8,67 €$ por $\mathrm{m}^{2}$, sendo a renda média do cluster de $9,43 € / \mathrm{m}^{2}$;

- Cluster 2 - Pena, São Mamede, Campolide, Mercês, Lapa, Campo Grande, N. Senhora Fátima, S. João de Deus, São Jorge de Arroios, Santos-o-Velho nas quais o valor da renda está compreendida entre $11,27 €$ e $10,29 € / \mathrm{m}^{2}$ e a renda média do cluster de $10,73 € / \mathrm{m}^{2}$;

- Cluster 3 - S. Sebastião Pedreira, Encarnação, Santa Isabel, S. Maria de Belém, S. Maria Olivais, Coração de Jesus e São José, nas quais o valor da renda está compreendida entre $12,74 €$ e $11,52 € / \mathrm{m} 2$ e a renda média do cluster de $12,08 € / \mathrm{m} 2$.

As diferenças entre os clusters 1, 2 e 3 são estatisticamente significativas a 99\%.

De referir que o desvio padrão dos T0_T1 é mais elevado que o dos T2, sinal de que as rendas da primeira tipologia apresentam um risco mais elevado.

Os Mapas 1 e 2 representam os clusters das rendas dos T0_T1 e T2, respetivamente. É observável que apesar de haver diferenças na hierarquia das freguesias dos T0_T1 para os $\mathrm{T} 2$, os lugares centrais apresentam valores mais elevados. 
Mapa 1 - Localização das freguesias das rendas do concelho de Lisboa (Rendas T0_T1)

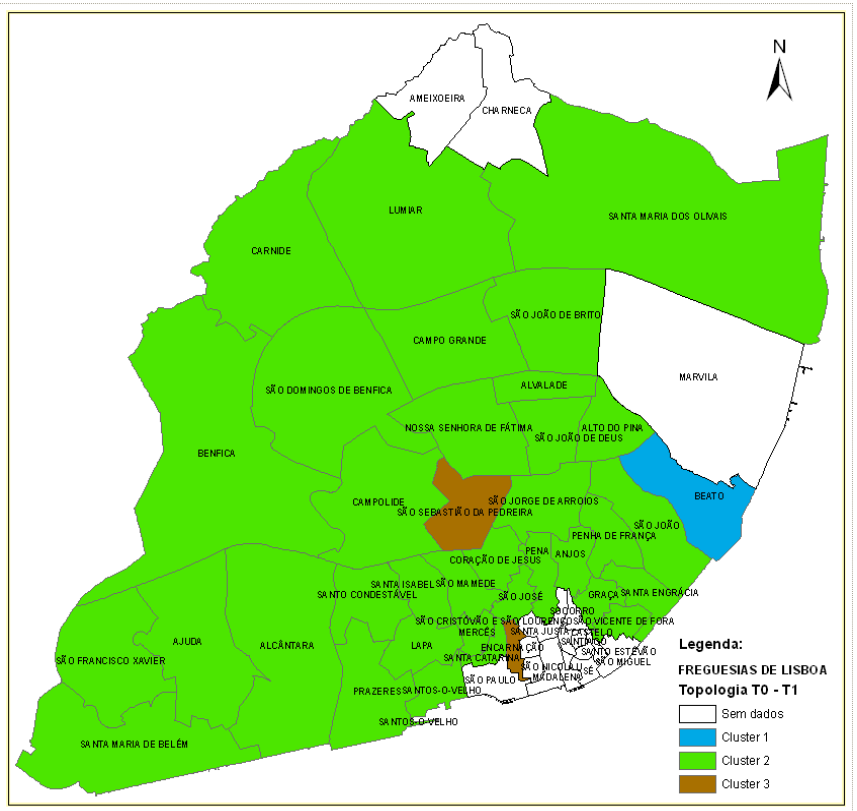

Mapa 2 - Localização das freguesias das rendas do concelho de Lisboa (Rendas T2)

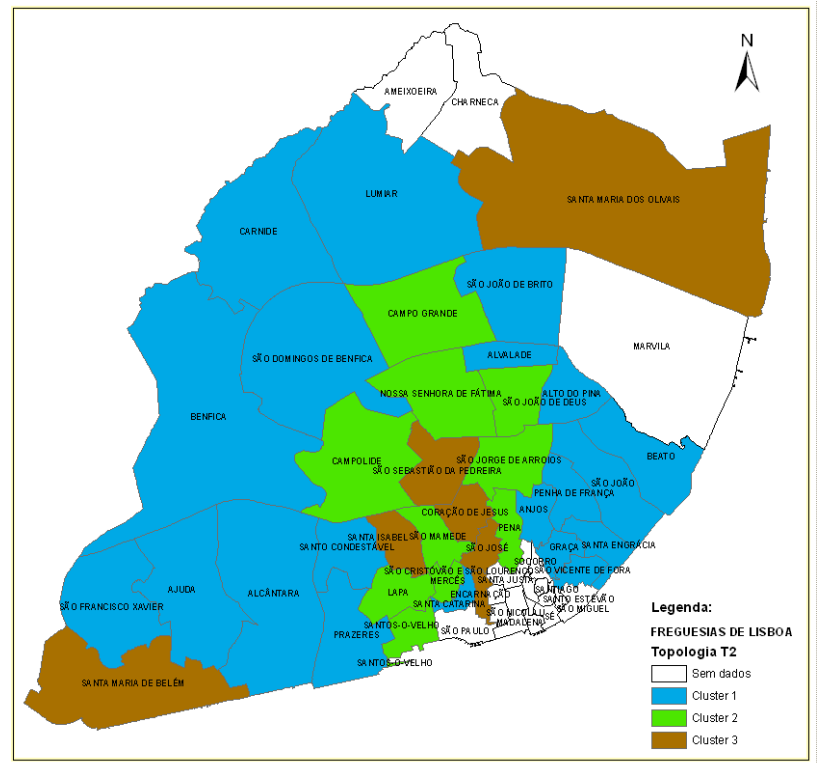

O Quadro 4 apresenta as yields médias das freguesias de Lisboa. Como esta análise de clusters serve de base para construir os modelos de regressão, foram retiradas as freguesias do concelho de Lisboa que apresentavam um desvio padrão nas rendas superior a 1,5, pois devido ao impacto que tinham nos resíduos das regressões, estas freguesias não permitiam apresentar os modelos como tendo um bom ajustamento. Na tipologia T0 e T1 a freguesia com o valor mais elevado é a de S. Maria dos Olivais com valor de yield superior a 6,00\%. As freguesias de Santos-o-Velho, São João de Brito, São Francisco Xavier e Alvalade apresentam os valores mais baixos de yields e inferiores a 5,00\%. Na tipologia T2 a freguesia de S. Maria do Olivais é a que apresenta o valor da yield mais 
elevado e superior a $6,00 \%$. Os valores mais baixos nesta tipologia são apresentados pelas freguesias de Alcântara e Campo Grande. Nas freguesias do concelho de Lisboa as yields médias dos T0 e T1 (5,38\%) são mais elevados que as yields médias dos T2 (5,12\%) e ambas inferiores às yields médias dos concelhos da amostra apresentada. Para as Tipologias T0 e T1, a análise de clusters hierárquica efetuada às yields das freguesias do concelho de Lisboa apresenta os mesmos resultados (3 clusters) pelo método do complete linkage ${ }^{3}$ usando a distância euclidiana quadrática como medida de dissemelhança entre sujeitos, conforme é possível observar no Quadro 4.

\begin{tabular}{|c|c|c|c|c|c|c|c|c|c|}
\hline \multicolumn{5}{|c|}{ Freguesias de Lisboa - Tipologias T0 e T1 } & \multicolumn{5}{|c|}{ Freguesias de Lisboa - Tipologias T2 } \\
\hline & $\begin{array}{c}\text { Yield } \\
\text { Média }\end{array}$ & $\begin{array}{l}\text { Desvio } \\
\text { Padrão }\end{array}$ & Cluster & $\begin{array}{c}\text { Yield } \\
\text { Média do } \\
\text { Cluster }\end{array}$ & & $\begin{array}{c}\text { Yield } \\
\text { Média }\end{array}$ & $\begin{array}{l}\text { Desvio } \\
\text { Padrão }\end{array}$ & Cluster & $\begin{array}{c}\text { Yield } \\
\text { Média do } \\
\text { Cluster }\end{array}$ \\
\hline S. Maria dos Olivais & 6,01 & 0,47 & \multirow{4}{*}{5,86} & \multirow{4}{*}{3} & S. M. Olivais & 6,04 & 0,53 & \multirow{6}{*}{5,67} & \multirow{6}{*}{3} \\
\hline Mercês & 5,85 & 0,71 & & & S. M. Belém & 5,73 & 0,37 & & \\
\hline Penha de França & 5,81 & 0,33 & & & Campolide & 5,69 & 0,74 & & \\
\hline Prazeres & 5,75 & 0,54 & & & Beato & 5,59 & 0,48 & & \\
\hline Campolide & 5,66 & 0,52 & \multirow{14}{*}{5,44} & \multirow{14}{*}{2} & Benfica & 5,54 & 0,36 & & \\
\hline Benfica & 5,60 & 0,44 & & & Santa Engrácia & 5,46 & 0,56 & & \\
\hline Lumiar & 5,58 & 0,38 & & & Mercês & 5,34 & 0,74 & \multirow{8}{*}{5,11} & \multirow{8}{*}{2} \\
\hline Alcântara & 5,56 & 0,57 & & & Alto do Pina & 5,25 & 0,73 & & \\
\hline Santo Condestável & 5,48 & 0,43 & & & S. J. Arroios & 5,25 & 0,61 & & \\
\hline S. D. de Benfica & 5,47 & 0,31 & & & Lumiar & 5,18 & 0,36 & & \\
\hline Alto do Pina & 5,44 & 0,38 & & & Santos-o-Velho & 4,98 & 0,52 & & \\
\hline S. Jorge de Arroios & 5,44 & 0,56 & & & S. D. Benfica & 4,98 & 0,25 & & \\
\hline Santa Engrácia & 5,40 & 0,64 & & & Anjos & 4,96 & 0,67 & & \\
\hline Beato & 5,34 & 0,73 & & & S. J. Brito & 4,90 & 0,35 & & \\
\hline Campo Grande & 5,34 & 0,45 & & & Carnide & 4,89 & 0,39 & \multirow{10}{*}{4,80} & \multirow{10}{*}{1} \\
\hline Carnide & 5,32 & 0,39 & & & Prazeres & 4,88 & 0,60 & & \\
\hline N. S. de Fátima & 5,26 & 0,44 & & & Santo Condestável & 4,88 & 0,49 & & \\
\hline S. Maria de Belém & 5,26 & 0,68 & & & N. S. Fátima & 4,88 & 0,39 & & \\
\hline Anjos & 5,23 & 0,64 & \multirow{6}{*}{4,90} & \multirow{6}{*}{1} & Penha de França & 4,87 & 0,69 & & \\
\hline Lapa & 5,07 & 0,39 & & & Lapa & 4,79 & 0,35 & & \\
\hline Santos-o-Velho & 4,87 & 0,52 & & & S. F. Xavier & 4,77 & 0,41 & & \\
\hline S. João de Brito & 4,85 & 0,29 & & & Alvalade & 4,74 & 0,49 & & \\
\hline S. Francisco Xavier & 4,77 & 0,47 & & & Campo Grande & 4,71 & 0,44 & & \\
\hline Alvalade & 4,63 & 0,46 & & & Alcântara & 4,64 & 0,32 & & \\
\hline Média & 5,38 & 0,49 & & & Média & 5,12 & 0,49 & & \\
\hline
\end{tabular}

Fonte: Elaboração própria

Assim, formaram-se 3 clusters, sendo:

- Cluster 1 - Anjos, Lapa, Santos-o-Velho, S. João de Brito, S. Francisco Xavier e Alvalade com yields entre os $5,23 \%$ e os $4,63 \%$, sendo a yield média do cluster de $4,90 \%$;

- Cluster 2 - Campolide, Benfica, Lumiar, Alcântara, Santo Condestável, S. D. de Benfica, Alto do Pina, S. Jorge de Arroios, Santa Engrácia, Beato, Campo Grande,

\footnotetext{
${ }^{3}$ Medida de Distância ou dissemelhança em que a distância entre 2 casos (a e b) é a raiz quadrada do somatório dos quadrados das diferenças entre os valores a e b para todas as variáveis (Reis, 2001).
} 
Carnide, N. S. de Fátima, S. Maria de Belém com yields entre os 5,66\% e os 5,26\%, sendo a yield média do cluster de 5,44\%;

- Cluster 3-S. Maria dos Olivais, Mercês, Penha de França e Prazeres com yields entre os $6,01 \%$ e os $5,75 \%$, sendo a yield média do cluster de $5,86 \%$.

As diferenças entre os clusters 1, 2 e 3 são estatisticamente significativas a 99\%.

Para as Tipologias T2, a análise hierárquica de clusters às yields das freguesias do concelho de Lisboa apresenta 3 clusters pelo método da maior distância (complete linkage - furthest neighbor) e a distância euclidiana como medida de dissemelhança métrica. A solução apresentada é observada no Quadro 4.

São formados 3 clusters:

- $\quad$ Cluster 1 - Carnide, Prazeres, Santo Condestável, N. S. Fátima, Penha de França, Lapa, S. F. Xavier, Alvalade, Campo Grande e Alcântara com yields entre os 4, $89 \%$ e os 4,64\%, apresentando o cluster uma yield média de 4,80\%;

- $\quad$ Cluster 2 - Mercês, Alto do Pina, S. J. Arroios, Lumiar, Santos-o-Velho, S. D. Benfica, Anjos e S. J. Brito com yields entre os $5,34 \%$ e os $4,90 \%$, sendo a yield média do cluster de $5,11 \%$;

- $\quad$ Cluster 3 -S. M. Olivais, S. M. Belém, Campolide, Beato, Benfica e Santa Engrácia com yields entre os $6,04 \%$ e os $5,46 \%$, sendo a yield média do cluster de $5,67 \%$.

As diferenças entre os clusters 1, 2 e 3 são estatisticamente significativas a 99\%.

Os Mapas 3 e 4 representam os clusters das yields dos T0_T1 e T2. Pode-se verificar que a localização dos clusters das yields dos T0_T1 é diferente da localização dos T2. Também por comparação com os Mapas 1 e 2 podemos verificar que a localização dos níveis de rendas e de yields são substancialmente diferentes. A uma renda elevada nem sempre corresponderá uma yield elevada.

Mapa 3 - Localização das freguesias das rendas do concelho de Lisboa (Yields T0_T1) 


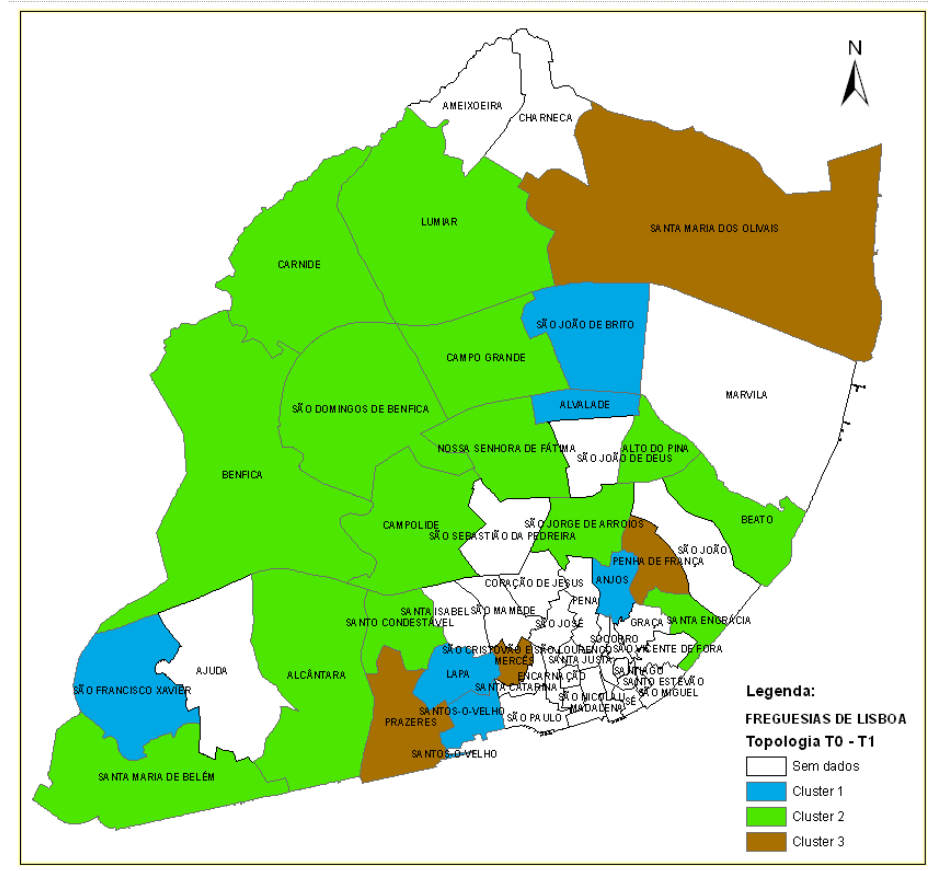

Mapa 4 - Localização das freguesias das rendas do concelho de Lisboa (Yields T2)

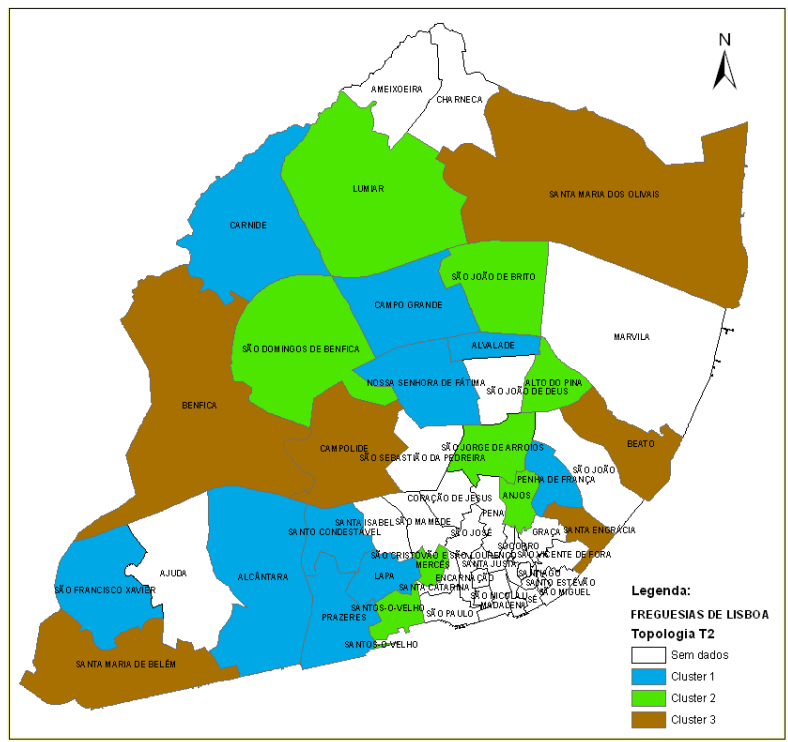

No Quadro 5 apresenta-se a regressão referente aos apartamentos T0_T1 das freguesias de Lisboa. Foram estudados os modelos de regressão para os valores por $\mathrm{m}^{2}$ dos T0_T1 e para os T2. Dentro destas tipologias foram estudadas as regressões para o conjunto das séries dos concelhos anteriormente apresentados.

Podemos verificar que o modelo para os $\mathrm{T} 0 \_\mathrm{T} 1$ é robusto. No modelo o valor por $\mathrm{m}^{2} \mathrm{da}$ oferta é dado por:

\section{Modelo:}


Valor_M ${ }^{2}$ Oferta_T0_T1 $=2004,577+$ 0,349*Avaliação_T0_T1 - 291,101* Cluster2_YieldT0_T1 -350,173*Cluster3_YieldT0_T1 - 0,004*DensidadePopulacional Neste modelo, as variáveis Cluster2YieldT0_T1 (dummy) e Cluster3YieldT0_T1 (dummy) têm por base o cluster 1 das yields dos T0_T1. Assim, o cluster 2 das yields tem uma desvalorização de 291,101€ relativamente ao cluster 1 das yields dos T0_T1 e o o cluster3 das yields uma desvalorização de 350,173€. Atendendo ao valor da estatística de Durbin-Watson $(0,628)$ pode-se afirmar que poderá ser utilizado o modelo de regressão apresentado para fazer previsões. De acordo com os valores da estatística F, é possível afirmar que existe uma relação linear explicada pelo modelo. Verifica-se que os coeficientes de regressão não apresentam colinearidade. Usando a variável dos resíduos gerados, o teste de ajustamento de Kolmogorov-Smirnov com a correção de Lilliefors é de 0,200. Assim, para o teste de Kolmogorov-Smirnov (amostra>50) é superior ao nível de significância 0,05 , pelo que não se rejeita a hipótese da normalidade dos resíduos.

\begin{tabular}{l} 
Quadro 5 - Modelos de regressão das tipologias T0_T1 com dados das freguesias de Lisboa \\
$\qquad$\begin{tabular}{|l|r|r|}
\hline & Modelo & sig \\
\hline Constante & 2004,577 & 0,000 \\
\hline AvaliaçãoT0_T1 & 0,349 & 0,000 \\
\hline Cluster2YieldT0T1 & $-291,101$ & 0,000 \\
\hline Cluster3YieldT0T1 & $-350,173$ & 0,000 \\
\hline Densidade Populacional & $-0,004$ & 0,004 \\
\hline $\mathrm{R}$ & 0,710 & \\
\hline $\mathrm{R}^{2}$ & 0,505 & \\
\hline $\mathrm{R}_{\mathrm{a}}{ }_{\mathrm{a}}$ & 0,499 & \\
\hline Durbin-Watson & 0,628 & \\
\hline $\mathrm{F}$ & 96,529 & 0,000 \\
\hline
\end{tabular} \\
\hline
\end{tabular}

No Quadro 6 pode ser observada a regressão referente aos apartamentos da tipologia T2 nas freguesias de Lisboa. No modelo o valor por $\mathrm{m}^{2}$ da oferta é dado por:

\section{Modelo:}

Valor_M ${ }^{2}$ Oferta_T2 $=1715,144+0,368 *$ Avaliação_T2 $-155,632 *$ Cluster2_YieldT2 -357,468*Cluster3_YieldT2 + 0,007*DensidadePopulacional

Neste modelo as variáveis Cluster2YieldT2 (dummy) e Cluster3YieldT2 (dummy) têm por base o cluster 1 das yields dos T2. Assim, o cluster2 das yields tem uma desvalorização de 155,632€ relativamente ao cluster 1 das yields dos T2 e o cluster 3 das yields uma desvalorização de $357,468 €$. Atendendo ao valor da estatística de Durbin- 
Watson $(0,628)$ pode-se afirmar que poderá ser utilizado o modelo de regressão apresentado para fazer previsões.

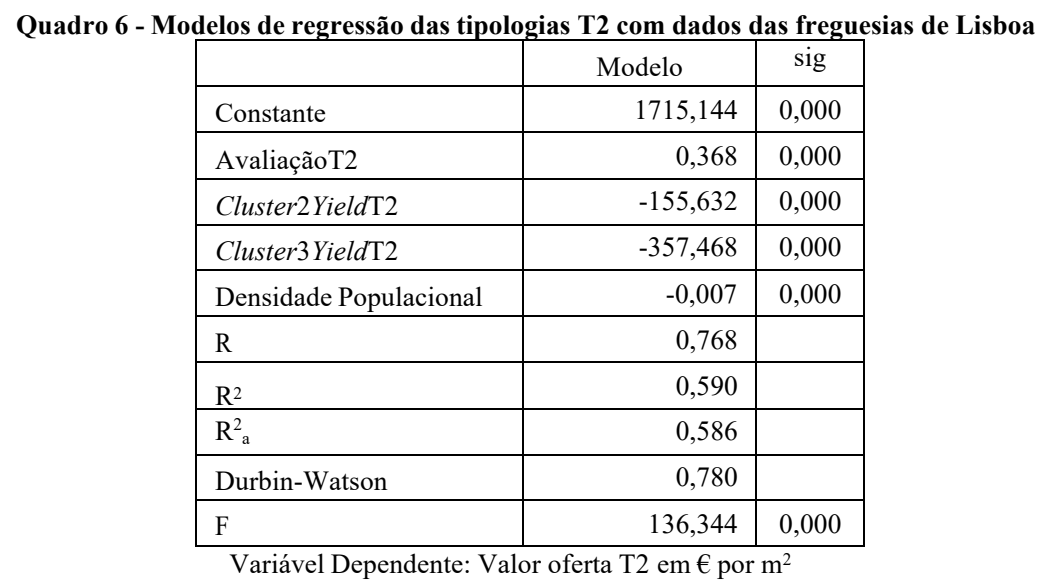

De acordo com os valores da estatística $\mathrm{F}$, é possível afirmar que existe uma relação linear explicada pelo modelo. Verifica-se que os coeficientes de regressão não apresentam colinearidade. Usando a variável dos resíduos gerados, o teste de ajustamento de Kolmogorov-Smirnov com a correção de Lilliefors é de 0,200. Assim, para o teste de Kolmogorov-Smirnov (amostra $>50$ ) é superior ao nível de significância 0,05, pelo que não se rejeita a hipótese da normalidade dos resíduos.

\section{5 - Conclusões}

O investidor espera que os mercados imobiliários tenham um comportamento idêntico aos mercados financeiros. Apesar de, no curto prazo, qualquer mercado poder apresentar bolhas especulativas e mispricing, espera-se que no médio e longo prazo o mercado seja racional e apresente um retorno naturalmente associado ao risco, de acordo com o enunciado por Markowitz. Dessa forma, os rendimentos gerados pela propriedade imobiliária estão na base do seu modelo de avaliação - o modelo do rendimento.

Neste artigo apresentou-se uma revisão da literatura sobre o estado da arte na utilização do modelo do rendimento como paradigma de avaliação imobiliária e procurou-se analisar a situação do mercado imobiliário de Lisboa.

Para o investidor interessa a análise do nível que as rendas podem atingir mas, mais importante, ainda será o nível das yields. Nas séries estudadas, os clusters de rendas são distintos dos clusters das yields e as freguesias com as yields mais elevadas são distintas das freguesias com o nível de renda mais elevado. Conclui-se, portanto, que o investidor 
necessita de estar atento às oportunidades de mercado, onde é pertinente que a avaliação imobiliária esteja em consonância com a evolução do mercado que se pretende cada vez mais eficiente, em que os valores fundamentais são essenciais e tendem a ser mais importantes que os valores sentimentais.

Nas freguesias de Lisboa, para se constituírem os clusters das yields foram retiradas as freguesias que nos valores de renda por $\mathrm{m}^{2}$ apresentavam um desvio padrão superior a 1,5. Isto deve-se à necessidade de constituir uma série estável, com baixa variância a fim de construir um bom modelo de regressão. As freguesias com a variância mais elevada apresentam naturalmente maior risco.

Através das regressões ficou explicado de forma cabal que o valor da oferta (variável dependente) é explicado pelas variáveis independentes da avaliação pelo método do rendimento, pelo cluster das yields e pela densidade populacional. Estas três variáveis independentes dão robustez aos seis modelos apresentados.

$\mathrm{Na}$ análise do mercado imobiliário é importante para o investidor racional conhecer as yields médias das diferentes zonas e como elas se agrupam em termos económicofinanceiros e geográficos.

\section{6 - Limitações do Estudo}

Depois da recolha de dados houve alterações na denominação e configuração do espaço geográfico das freguesias do concelho de Lisboa. Esta alteração não coloca em causa a cientificidade do estudo, mas indicia uma clara necessidade de atualização. Seria também interessante para estudos posteriores, analisar a evolução do mercado ao longo dos anos, e verificar qual o impacto nas rendas e yields do mercado habitacional, no concelho de Lisboa, da crise do subprime.

\section{Bibliografia}

- $\quad$ Black, A., Fraser, P. \& Hoesli, M. (2006). House Prices, Fundamentals and Bubbles. Journal of Business Finance and Accounting, 33 (9, 10), 1535-1555.

- Boyd, T. (2002). Property cash flow studies: focusing on model consistency and data accuracy, Working Paper, Queensland University of Technology, Brisbane.

- Brown, G. \& Cliff, M. (2005). Investor Sentiment and Asset Valuation. Journal of Business, 78(2), 405- 440. 
- Campbell, J. (1987). Does Saving Anticipate Declining Labor Income? An Alternative Test of the Permanent Income Hypothesis. Econometrica, 55, 1249-1273.

- Campbell, J. \& Shiller, R. (1987). Cointegration and Tests of Present Value Models. Journal of Political Economy, 95, 1062-1088.

- Clayton, J., Ling, D. C. \& Naranjo, A. (2009). Commercial Real Estate Valuation: Fundamentals Versus Investor Sentiment. Journal The Journal of Real Estate Finance and Economics, 38(1), 5-37.

- $\quad$ De Long, Bradford, Shleifer, A., Summers, L.H. \& Waldmann, R.J. (1990). Noise Trader Risk in Financial Markets. Journal of Political Economy, 98(4), 703-738.

- Deng, Y., Gabriel, S. A. \& Nothaft, F. (2002). Duration of Residence in the Rental Housing Market. USC FBE Working Paper No. 02-3. Available at SSRN: http://ssrn.com/abstract=304641 or DOI: $10.2139 /$ ssrn.10.2139/ssrn.304641

- French, N. (2004). The Valuation of Specialised Property - A review of valuation methods. Journal of Property Investment \& Finance, 22 (6), 533-541.

- Gallin, J. (2003). The Long-Run Relationship between House Prices and Income: Evidence from Local Housing Markets. Working Paper, Federal Reserve Board.

- Geltner, D., Miller, N., Clayton, J. \& Eicholtz, P. (2007). Commercial Real Estate Analysis and Investments. 2nd Edition, South-Western Publishing.

- Ghysels, E., Plazzi, A. \& Valkanov, R. (2006). Valuation in the US Commercial Real Estate. Working Paper resultado direto da Conferência no EFMA, Madrid, 2006.

- Gordon, M. J. \& Shapiro, E. (1956). Capital Equipment Analysis: The required rate of profit. Management Science, 3, 102-110.

- Graham, B. \& Dodd, D. (1934). Security Analysis. New York: McGraw-Hill.

- Grenadier, S. R. (2003). An Equilibrium Analysis of Real Estate Leases. Working Paper 9475, National Bureau of Economic Research.

- Gujarati, D. N. (2003). Basic Econometrics. Fourth Edition, Mc Graw Hill, International Edition.

- Ho, K., Rengarajan, S., \& Glascock, J. (2014). An Examination of the Structure and Dynamics of Singapore's Maturing Central Area Office Market. Journal of Property Investment \& Finance, 32(5), 485-504.

- Hordijk, A. \& Ridder, W. (2005). Valuation Model Uniformity and Consistency in Real Estate Indices - The case of The Netherlands. Journal of Property Investment \& Finance, 23(2), 165-181. 
- Laia, A. N. (2007). Avaliação de Imóveis pelo Método da Cap rate ou Yield. Revista Confidencial Imobiliário, (Abril), pp. 29-30.

- León, E. M. (2003). Valoración Inmobiliaria - Estúdio y cálculo del valor de los bienes inmuebles de natureza urbana, y de determinados derechos. Dykson. Madrid.

- Ling, D. \& Archer, W. (2006). Real Estate Principles: A Value Approach. McGrawHill, Irwin, USA.

- Lintner, J. (1965). The Valuation of Risk Assets and the Selection of Risky Investments in Stock Portfolios and Capital Budgets. Review of Economics and Statistics, 47(1), 13-37.

- Lusht, K.M. (2001). Real Estate Valuation: Principles and Applications. KML, State College, PA.

- Markowitz, H. (1959). Portfolio Selection: Efficient diversification of investments. New York: Wiley.

- Markowitz, H. (1952). Portfolio Selection. Journal of Finance, 7(1), 77-91

- Maroco, J. (2014). Análise Estatística com o SPSS Statistics. 6. ed. Lisboa: ReportNumber.

- Meese, R. \& Wallace, N. (1993). Testing the Present Value Relation For Housing Prices: Should I Leave My House in San Francisco?. Journal of Urban Economics, $35,245-266$.

- Molina, M.-G. A. (2003). Valoración Inmobiliária. Editorial Montecorvo, S.A..

- Moreira, A. C., Tavares, F. O., \& Pereira, E. T. (2014). Valoración Inmobiliaria. Aplicación do método de rendas nos municípios portugueses. Revista Galega de Economía, 23(1), 187-202.

- Mossin, J. (1966). Equilibrium in a Capital Asset Market. Econometrica, 34(4),768783.

- Pagliari, J.L. Jr (1991). Inside the real estate yield. Real Estate Review, 21(3), 48-53.

- Pestana, M. H. \& Gageiro, J. N. (2014). Análise de Dados para Ciências Sociais: A Complementaridade do SPSS. Edições Sílabo, $6^{\text {a }}$ Edição Revista e aumentada, Lisboa.

- Rebelo, E. (2002). Mercado Imobiliário e Transformações Urbanas. Tese de Doutoramento em Engenharia Civil, submetida à Faculdade de Engenharia da Universidade do Porto.

- Reis, E. (2001). Estatística Multivariada Aplicada. Lisboa: Edições Sílabo. 
- Scolese, D., Bergmann, D. R., da Silva, F. L., \& Savoia, J. R. F. (2015). Análise de Estilo de Fundos Imobiliários no Brasil. Revista de Contabilidade $e$ Organizações, 9(23), 24-35.

- Sharafeddine, R. I. (2015). A Cash-Flow Theory of Stock Valuation. International Journal of Finance and Accounting, 4(1), 79-107.

- Sharpe, W. F. (1964). Capital Assets prices: a theory of market equilibrium under conditions of risk. Journal of Finance, 19(3), 425-442.

- Tabner, I. T. (2007). Predicting House Prices in Perpetuity when Greed, Fear and Value Expression vie with Fundamentals. Working Paper, Department of Accounting and Finance, University of Stirling, Scotland FK9 4LA.

- Tavares, F. \& Almeida, L. (2015). O setor do Imobiliário Residencial em Portugal de 2000 a 2013. E3 - Revista de Economia, Empresas e Empreendedores na CPLP, 2(2), 4-26.

- Tavares, Fernando, Moreira, A. C. \& Pereira, E. T. (2011). Método do rendimento na avaliação imobiliária: uma revisão da literatura. Economia Global e Gestão, 16(2), $37-61$.

- Van Gool, P., Jager, P. \& Weisz, R.M. (2001). Onroerend Goed als Belegging, Stenfert Kroese, Culemborg.

- Wang, K. \& Zhou, Y. (2000). Overbuilding: A game-theoretic approach. Real Estate Economics, 28(3), 493-522. 